\title{
Single-top and top-antitop cross sections
}

\author{
Nikolaos Kidonakis ${ }^{1, *}$ \\ ${ }^{1}$ Department of Physics, Kennesaw State University, Kennesaw, GA 30144, USA
}

\begin{abstract}
I present high-order calculations, including soft-gluon corrections, for single-top and top-antitop production cross sections and differential distributions. For single-top production, results are presented for the three different channels in the Standard Model, for associated production with a charged Higgs, and for processes involving anomalous couplings. For top-antitop pair production, total cross sections and top-quark transverse-momentum and rapidity distributions are presented for various LHC energies.
\end{abstract}

\section{Introduction}

Higher-order soft-gluon corrections have been calculated through $\mathrm{N}^{3} \mathrm{LO}$ for top-quark production via various processes; see Ref. [1] for a review. Here I present the latest results for $t$-channel and $s$-channel single-top production, $t W$ and $t H^{-}$production, $t Z$ production via anomalous couplings, and $t \bar{t}$ production. For all these processes QCD corrections are very significant and are dominated by soft-gluon corrections.

I calculate and resum these soft corrections at next-to-next-to-leading logarithm (NNLL) accuracy for the double-differential cross section, which is then used to calculate top-quark transverse-momentum and rapidity distributions and total cross sections. Finite-order expansions at approximate NNLO (aNNLO) and approximate $\mathrm{N}^{3} \mathrm{LO}\left(\mathrm{aN}^{3} \mathrm{LO}\right)$, matched to exact results, provide the best predictions for these quantities.

We calculate soft-gluon corrections for partonic processes of the form

$$
f_{1}\left(p_{1}\right)+f_{2}\left(p_{2}\right) \rightarrow t\left(p_{t}\right)+X
$$

and we define $s=\left(p_{1}+p_{2}\right)^{2}, t=\left(p_{1}-p_{t}\right)^{2}, u=\left(p_{2}-p_{t}\right)^{2}$, and $s_{4}=s+t+u-\sum m^{2}$. At partonic threshold $s_{4} \rightarrow 0$. The $n$ th-order soft-gluon corrections appear as $\left[\ln ^{k}\left(s_{4} / m_{t}^{2}\right) / s_{4}\right]_{+}$ where $k \leq 2 n-1$.

Moments of the partonic cross section, $\hat{\sigma}(N)=\int\left(d s_{4} / s\right) e^{-N s_{4} / s} \hat{\sigma}\left(s_{4}\right)$, can be written in factorized form

$$
\sigma^{f_{1} f_{2} \rightarrow t X}(N, \epsilon)=H_{I L}^{f_{1} f_{2} \rightarrow t X}\left(\alpha_{s}\left(\mu_{R}\right)\right) S_{L I}^{f_{1} f_{2} \rightarrow t X}\left(\frac{m_{t}}{N \mu_{F}}, \alpha_{s}\left(\mu_{R}\right)\right) \prod J_{\text {in }}\left(N, \mu_{F}, \epsilon\right) \prod J_{\text {out }}\left(N, \mu_{F}, \epsilon\right)
$$

in $4-\epsilon$ dimensions, where $H_{I L}^{f_{1} f_{2} \rightarrow t X}$ is a hard function and $S_{L I}^{f_{1} f_{2} \rightarrow t X}$ is a soft-gluon function. $S_{L I}^{f_{1} f_{2} \rightarrow t X}$ satisfies the renormalization group equation

$$
\left(\mu \frac{\partial}{\partial \mu}+\beta\left(g_{s}\right) \frac{\partial}{\partial g_{s}}\right) S_{L I}^{f_{1} f_{2} \rightarrow t X}=-\left(\Gamma_{S}^{\dagger}\right)_{L K}^{f_{1} f_{2} \rightarrow t X} S_{K I}^{f_{1} f_{2} \rightarrow t X}-S_{L K}^{f_{1} f_{2} \rightarrow t X}\left(\Gamma_{S}\right)_{K I}^{f_{1} f_{2} \rightarrow t X}
$$

*e-mail: nkidonak@kennesaw.edu 
where the soft anomalous dimension $\Gamma_{S}^{f_{1} f_{2} \rightarrow t X}$ controls the evolution of the soft function, giving the exponentiation of logarithms of $N$. To achieve NNLL accuracy we need to calculate the relevant soft anomalous dimensions at two loops.

\section{Single-top production}

We now provide results for single-top production in the $t$-channel, $s$-channel, and via $t W$ production. Fixed-order results for these processes are known at NNLO for the $t$-channel [2-4] and $s$-channel [5], and at NLO for $t W$ production [6]. Here we provide results with soft-gluon corrections at aNNLO for $t$ - and $s$-channel production [7], and at $\mathrm{aN}^{3} \mathrm{LO}$ for $t W$ production [8]; these results are obtained from NNLL resummation [7-9]. We use MMHT2014 NNLO pdf [10].
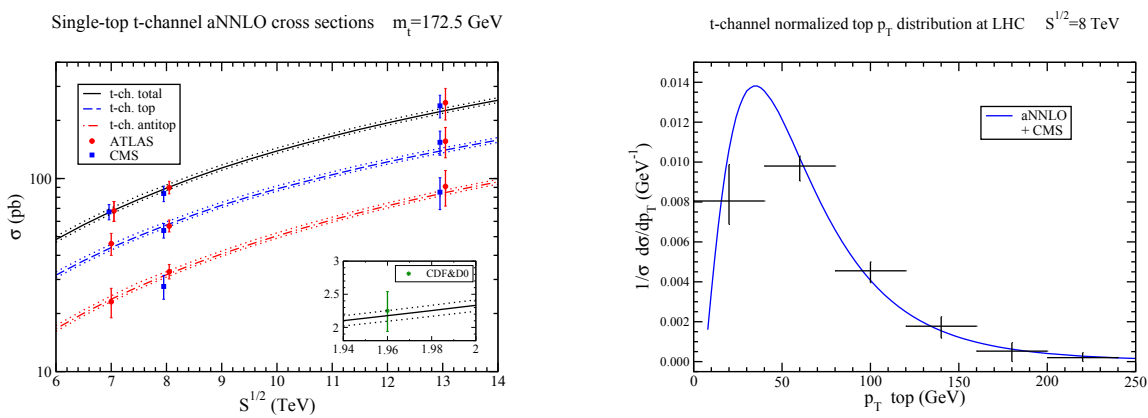

Figure 1. (Left) Single-top $t$-channel aNNLO cross sections compared with CMS and ATLAS data at $7 \mathrm{TeV}[12,13], 8 \mathrm{TeV}[14,15]$, and $13 \mathrm{TeV}[16,17]$, and with CDF and D0 combined data at 1.96 $\mathrm{TeV}$ [18]. (Right) Top-quark aNNLO normalized $p_{T}$ distributions in $t$-channel production at $8 \mathrm{TeV}$ compared to CMS [19] data.

Single-top s-channel aNNLO cross sections $\mathrm{m}_{\mathrm{t}}=172.5 \mathrm{GeV}$

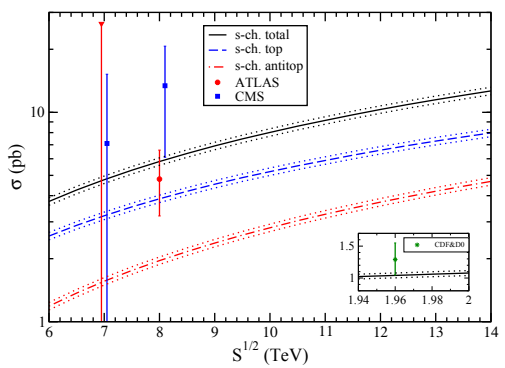

$\mathrm{tW}^{-}+\overline{\mathrm{tW}}^{+} \mathrm{aN}^{3} \mathrm{LO}$ cross section $\mathrm{m}_{\mathrm{t}}=172.5 \mathrm{GeV}$

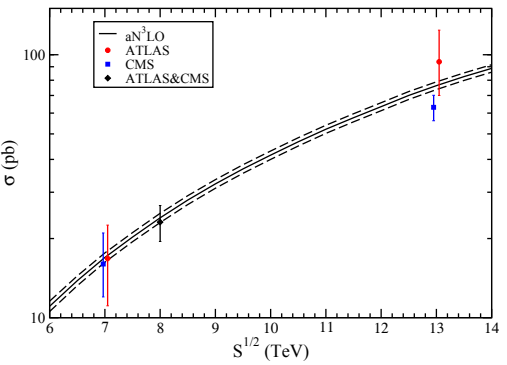

Figure 2. (Left) Single-top $s$-channel aNNLO cross sections compared to ATLAS and CMS data at 7 $\mathrm{TeV}[20,21]$ and $8 \mathrm{TeV}$ [21, 22], and to CDF and D0 combined data [23] at $1.96 \mathrm{TeV}$. (Right) $\mathrm{aN}^{3} \mathrm{LO}$ cross sections for $t W$ production compared to ATLAS and CMS data at $7 \mathrm{TeV}$ [24, 25], $8 \mathrm{TeV}$ [26], and $13 \mathrm{TeV}[27,28]$.

We begin with $t$-channel production at aNNLO. In the left plot of Fig. 1 we display total $t$-channel cross sections as functions of collider energy at the LHC and (inset) at the Tevatron. 
Results are given for the single-top cross section, the single-antitop cross section, and their sum. We observe very good agreement of the aNNLO theory curves $[7,11]$ with all available data from the LHC and the Tevatron at various energies. The right plot of Fig. 1 shows the aNNLO normalized top-quark $p_{T}$ distributions at $8 \mathrm{TeV}$ LHC energy, which describe the corresponding data from CMS [19] quite well.

We continue with $s$-channel production at aNNLO. In the left plot of Fig. 2 we show cross sections for single-top and single-antitop s-channel production, and their sum, as functions of energy at the LHC and (inset) at the Tevatron. We again observe very good agreement of the aNNLO theory curves $[7,11]$ with available data at LHC and Tevatron energies.

We next discuss $t W$ production at $\mathrm{aN}^{3} \mathrm{LO}$. In the right plot of Fig. 2 we show the total $t W^{-}+\bar{t} W^{+}$cross section as a function of LHC energy. Very good agreement is observed between the $\mathrm{aN}^{3} \mathrm{LO}$ results $[8,11]$ and the data at 7,8 , and $13 \mathrm{TeV}$ energies.

\section{$3 t H^{-}$production}

We continue with $t H^{-}$production in the MSSM or other two-Higgs-doublet models [29]. We use MMHT2014 NNLO pdf [10] for our numerical results.
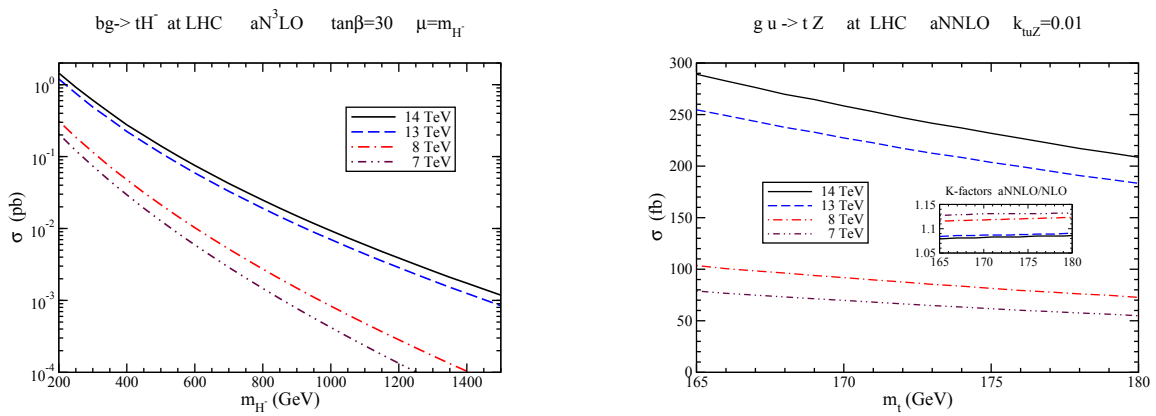

Figure 3. (Left) $\mathrm{aN}^{3} \mathrm{LO}$ cross sections for $t H^{-}$production. (Right) aNNLO cross sections for $t Z$ production via anomalous couplings.

In the left plot of Fig. 3 we show the $\mathrm{aN}^{3} \mathrm{LO}$ total cross section for $t H^{-}$production [11] as a function of charged-Higgs mass at LHC energies of 7, 8, 13, and $14 \mathrm{TeV}$. We use $\tan \beta=30$. The soft-gluon corrections are large for this process. Top-quark $p_{T}$ and rapidity distributions in this process have also been presented in [29].

\section{$4 t Z$ production via anomalous couplings}

Next, we discuss soft-gluon corrections in $t Z$ production in models with anomalous $t-q-Z$ couplings [30, 31]. The NLO corrections for this process were calculated in Ref. [32]. The complete NLO corrections are very well approximated by the soft-gluon corrections at that order.

We use CT14 pdf [33] for our numerical results in this process. In the right plot of Fig. 3 we plot the aNNLO total cross section for $t Z$ production as a function of top-quark mass at LHC energies of 7, 8, 13, and $14 \mathrm{TeV}$.

The $K$-factors shown in the inset plot show that the aNNLO corrections are large and significantly enhance the NLO cross section, especially at lower energies. This is important 
in providing theoretical input to experimental limits on the couplings [34, 35]. Top-quark differential distributions in this process have been presented in Ref. [31].

Similar results have more recently been presented for $t \gamma$ production via anomalous couplings in Ref. [36].

\section{Top-antitop pair production}

Finally, we discuss top-antitop pair production [37,38]. The soft anomalous dimensions are $2 \times 2$ matrices for the $q \bar{q} \rightarrow t \bar{t}$ channel, and $3 \times 3$ matrices for the $g g \rightarrow t \bar{t}$ channel.

$\mathrm{pp}->\mathrm{t} \overline{\mathrm{t}}$ at LHC energies $\mathrm{aN}^{3} \mathrm{LO} \quad \mathrm{m}_{\mathrm{t}}=172.5 \mathrm{GeV}$

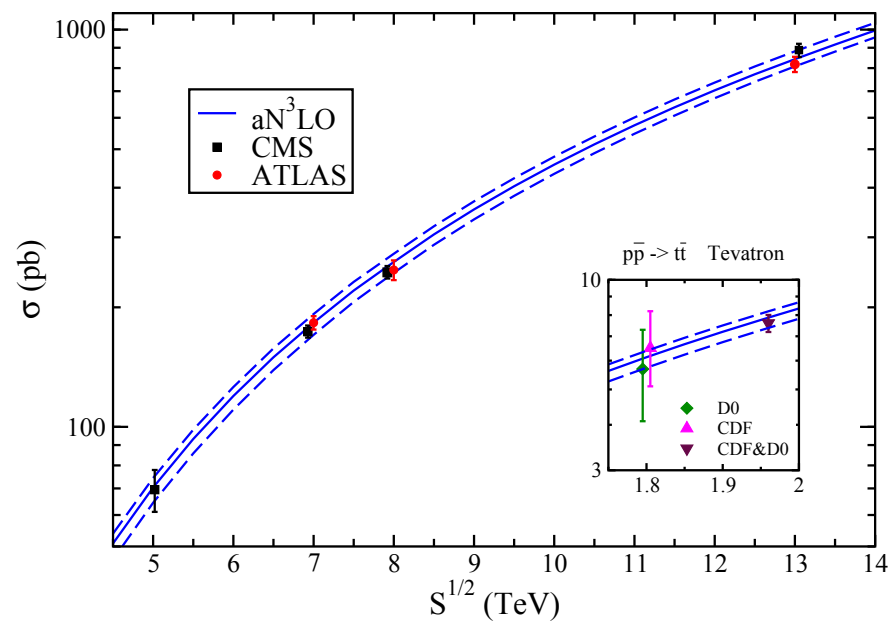

Figure 4. Top-antitop $\mathrm{aN}^{3} \mathrm{LO}$ cross sections compared with CMS data at $5.02 \mathrm{TeV}$ [41] and with ATLAS and CMS data at $7 \mathrm{TeV}[42,43], 8 \mathrm{TeV}[43,44]$, and $13 \mathrm{TeV}[45,46] \mathrm{LHC}$ energies. The inset shows the $\mathrm{aN}^{3} \mathrm{LO}$ cross section compared with CDF [47] and D0 [48] data at $1.8 \mathrm{TeV}$, and CDF\&D0 combined data [49] at $1.96 \mathrm{TeV}$ Tevatron energy.

At one loop for $q \bar{q} \rightarrow t \bar{t}$, the elements of the $\Gamma_{S}^{q \bar{q} \rightarrow t \bar{t}}$ matrix in an $s$-channel singlet-octet color basis are

$$
\begin{aligned}
& \Gamma_{11}^{q \bar{q} \rightarrow t \bar{t}(1)}=\Gamma_{\text {cusp }}^{(1)}, \quad \Gamma_{12}^{q \bar{q} \rightarrow t \bar{t}(1)}=\frac{C_{F}}{C_{A}} \ln \left(\frac{t_{1}}{u_{1}}\right), \quad \Gamma_{21}^{q \bar{q} \rightarrow t \bar{t}(1)}=2 \ln \left(\frac{t_{1}}{u_{1}}\right), \\
& \Gamma_{22}^{q \bar{q} \rightarrow t \bar{t}(1)}=\left(1-\frac{C_{A}}{2 C_{F}}\right) \Gamma_{\text {cusp }}^{(1)}+4 C_{F} \ln \left(\frac{t_{1}}{u_{1}}\right)-\frac{C_{A}}{2}\left[1+\ln \left(\frac{s m_{t}^{2} t_{1}^{2}}{u_{1}^{4}}\right)\right] .
\end{aligned}
$$

At two loops for $q \bar{q} \rightarrow t \bar{t}$, the elements of the $\Gamma_{S}^{q \bar{q} \rightarrow t \bar{t}}$ matrix are

$$
\begin{aligned}
\Gamma_{11}^{q \bar{q} \rightarrow t \bar{t}(2)} & =\Gamma_{\text {cusp }}^{(2)}, \quad \Gamma_{12}^{q \bar{q} \rightarrow t \bar{t}(2)}=\left(\frac{K}{2}-\frac{C_{A}}{2} N_{2 l}\right) \Gamma_{12}^{q \bar{q} \rightarrow t \bar{t}(1)}, \\
\Gamma_{21}^{q \bar{q} \rightarrow t \bar{t}(2)} & =\left(\frac{K}{2}+\frac{C_{A}}{2} N_{2 l}\right) \Gamma_{21}^{q \bar{q} \rightarrow t \bar{t}(1)}, \\
\Gamma_{22}^{q \bar{q} \rightarrow t \bar{t}(2)} & =\frac{K}{2} \Gamma_{22}^{q \bar{q} \rightarrow t \bar{t}(1)}+\left(1-\frac{C_{A}}{2 C_{F}}\right)\left(\Gamma_{\text {cusp }}^{(2)}-\frac{K}{2} \Gamma_{\text {cusp }}^{(1)}\right) .
\end{aligned}
$$


See also Ref. [1] for more details. Here $\Gamma_{\text {cusp }}$ is the cusp anomalous dimension [39].

The soft-gluon corrections are large and they are excellent approximations to the complete corrections at both NLO and NNLO. The additional corrections at $\mathrm{aN}^{3} \mathrm{LO}$ provide further significant enhancements and must be included for precision physics. Another approach for the $\mathrm{aN}^{3} \mathrm{LO}$ corrections has appeared recently in Ref. [40].

In our numerical results below we use the MMHT2014 NNLO pdf [10]. The total topantitop cross sections at $\mathrm{aN}^{3} \mathrm{LO}$ [38] are shown in Fig. 4 and compared with data at Tevatron and LHC energies. We find remarkable agreement between theory and data at all energies.
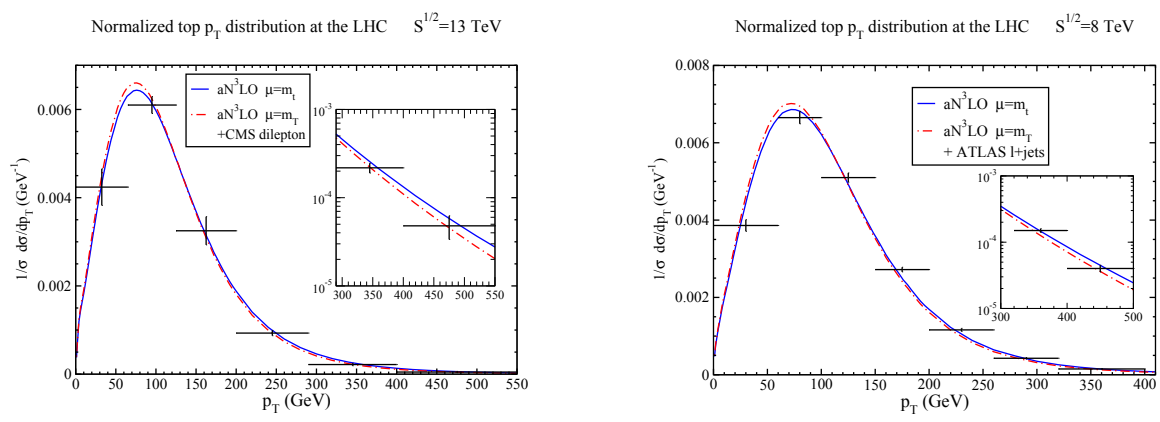

Figure 5. aN $\mathrm{N}^{3} \mathrm{LO}$ top-quark normalized $p_{T}$ distributions (left) at $13 \mathrm{TeV}$ energy compared with CMS [50] data and (right) at $8 \mathrm{TeV}$ energy compared with ATLAS [51] data.

The $\mathrm{aN}^{3} \mathrm{LO}$ top-quark normalized $p_{T}$ distributions in $t \bar{t}$ production are shown in Fig. 5 at $13 \mathrm{TeV}$ (left plot) and $8 \mathrm{TeV}$ (right plot) and compared with CMS and ATLAS data respectively. We find excellent agreement of the theoretical predictions with the data.
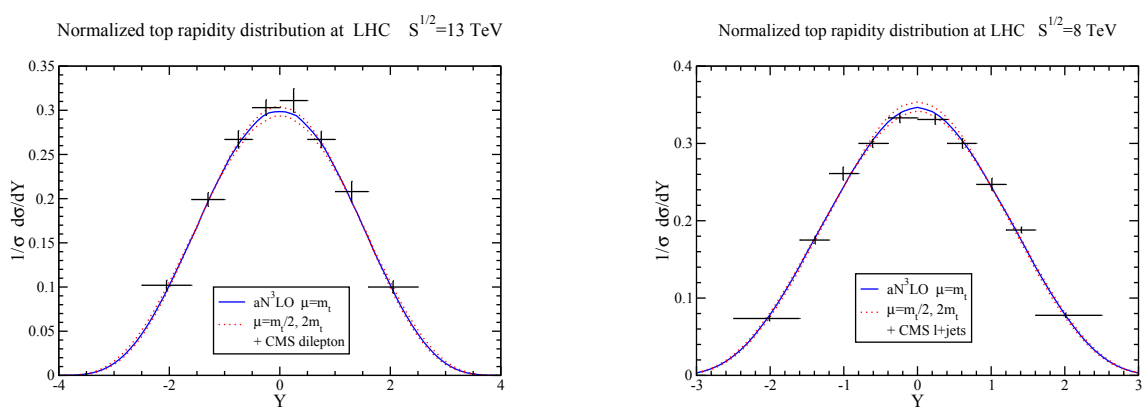

Figure 6. Top-quark $\mathrm{aN}^{3} \mathrm{LO}$ normalized rapidity distribution at (left) $13 \mathrm{TeV}$ energy compared with CMS data [50] and (right) $8 \mathrm{TeV}$ energy compared with CMS data [52].

The $\mathrm{aN}^{3} \mathrm{LO}$ top-quark normalized rapidity distributions in $t \bar{t}$ production are shown in Fig. 6 at $13 \mathrm{TeV}$ (left plot) and $8 \mathrm{TeV}$ (right plot) and compared with CMS data. Again, we find that the theory curves provide an excellent description of the data. 


\section{Summary}

We have discussed cross sections and distributions for various top-quark production processes. Soft-gluon corrections are important in all cases. We have shown results for $t$-channel and $s$-channel single-top production at aNNLO, $t W$ production at $\mathrm{aN}^{3} \mathrm{LO}, t H^{-}$production at $\mathrm{aN}^{3} \mathrm{LO}, t Z$ production via anomalous couplings at aNNLO, and $t \bar{t}$ production at $\mathrm{aN}^{3} \mathrm{LO}$. We find excellent agreement with available collider data. The higher-order corrections are very significant and need to be included for better theoretical predictions.

\section{Acknowledgments}

This material is based upon work supported by the National Science Foundation under Grant No. PHY 1519606.

\section{References}

[1] N. Kidonakis, Int. J. Mod. Phys. A 33, 1830021 (2018) [arXiv:1806.03336 [hep-ph]].

[2] M. Brucherseifer, F. Caola, and K. Melnikov, Phys. Lett. B 736, 58 (2014) [arXiv:1404.7116 [hep-ph]].

[3] E.L. Berger, J. Gao, C.-P. Yuan, and H.X. Zhu, Phys. Rev. D 94, 071501 (2016) [arXiv:1606.08463 [hep-ph]].

[4] E.L. Berger, J. Gao, and H.X. Zhu, JHEP 1711, 158 (2017) [arXiv:1708.09405 [hep-ph]].

[5] Z. Liu and J. Gao, arXiv:1807.03835 [hep-ph].

[6] S.H. Zhu, Phys. Lett. B 524, 283 (2002) [Erratum: ibid. 537, 351 (2002)] [hep$\mathrm{ph} / 0109269]$.

[7] N. Kidonakis, Phys. Rev. D 81, 054028 (2010) [arXiv:1001.5034 [hep-ph]]; 83, 091503(R) (2011) [arXiv:1103.2792 [hep-ph]]; 88, 031504(R) (2013) [arXiv:1306.3592 [hep-ph]]; 93, 054022 (2016) [arXiv:1510.06361 [hep-ph]].

[8] N. Kidonakis, Phys. Rev. D 96, 034014 (2017) [arXiv:1612.06426 [hep-ph]]; in Proceedings of DPF 2017, eConf C170731 [arXiv:1709.06975 [hep-ph]].

[9] N. Kidonakis, Phys. Rev. D 82, 054018 (2010) [arXiv:1005.4451 [hep-ph]].

[10] L.A. Harland-Lang, A.D. Martin, P. Molytinski, and R.S. Thorne, Eur. Phys. J. C 75, 204 (2015) [arXiv:1412.3989 [hep-ph]].

[11] N. Kidonakis, in Proceedings of CIPANP2018 [arXiv:1808.02934 [hep-ph]].

[12] CMS Collab., JHEP 1212, 035 (2012) [arXiv:1209.4533 [hep-ex]].

[13] ATLAS Collab., Phys. Rev. D 90, 112006 (2014) [arXiv:1406.7844 [hep-ex]].

[14] CMS Collab., JHEP 1406, 090 (2014) [arXiv:1403.7366 [hep-ex]].

[15] ATLAS Collab., Eur. Phys. J. C 77, 531 (2017) [arXiv:1702.02859 [hep-ex]].

[16] ATLAS Collab., JHEP 1704, 086 (2017) [arXiv:1609.03920 [hep-ex]].

[17] CMS Collab., Phys. Lett. B 772, 752 (2017) [arXiv:1610.00678 [hep-ex]].

[18] CDF and D0 Collab., Phys. Rev. Lett. 115, 152003 (2015) [arXiv:1503.05027 [hep-ex]].

[19] CMS Collab., CMS-PAS-TOP-14-004.

[20] ATLAS Collab., ATLAS-CONF-2011-118.

[21] CMS Collab., JHEP 1609, 027 (2016) [arXiv:1603.02555 [hep-ex]].

[22] ATLAS Collab., Phys. Lett. B 756, 228 (2016) [arXiv:1511.05980 [hep-ex]].

[23] CDF and D0 Collab., Phys. Rev. Lett. 112, 231803 (2014) [arXiv:1402.5126 [hep-ex]].

[24] ATLAS Collab., Phys. Lett. B 716, 142 (2012) [arXiv:1205.5764 [hep-ex]].

[25] CMS Collab., Phys. Rev. Lett. 110, 022003 (2013) [arXiv:1209.3489 [hep-ex]]. 
[26] ATLAS and CMS Collab., ATLAS-CONF-2016-023, CMS-PAS-TOP-15-019.

[27] ATLAS Collab., JHEP 1801, 063 (2018) [arXiv:1612.07231 [hep-ex]].

[28] CMS Collab., arXiv:1805.07399 [hep-ex].

[29] N. Kidonakis, JHEP 0505, 011 (2005) [hep-ph/0412422]; Phys. Rev. D 94, 014010 (2016) [arXiv:1605.00622 [hep-ph]].

[30] A. Belyaev and N. Kidonakis, Phys. Rev. D 65, 037501 (2002) [hep-ph/0102072]; N. Kidonakis and A. Belyaev, JHEP 0312, 004 (2003) [hep-ph/0310299].

[31] N. Kidonakis, Phys. Rev. D 97, 034028 (2018) [arXiv:1712.01144 [hep-ph]].

[32] B.H. Li, Y. Zhang, C.S. Li, J. Gao, and H.X. Zhu, Phys. Rev. D 83, 114049 (2011) [arXiv:1103.5122 [hep-ph]].

[33] S. Dulat, T.-J. Hou, J. Gao, M. Guzzi, J. Huston, P. Nadolsky, J. Pumplin, C. Schmidt, D. Stump, and C.-P. Yuan, Phys. Rev. D 93, 033006 (2016) [arXiv:1506.07443 [hep-ph]].

[34] CMS Collaboration, JHEP 1707, 003 (2017) [arXiv:1702.01404 [hep-ex]].

[35] ATLAS Collaboration, ATLAS-CONF-2017-070.

[36] M. Forslund and N. Kidonakis, arXiv:1808.09014 [hep-ph].

[37] N. Kidonakis, Phys. Rev. D 82, 114030 (2010) [arXiv:1009.4935 [hep-ph]]; in Physics of Heavy Quarks and Hadrons, HQ2013 [arXiv:1311.0283 [hep-ph]].

[38] N. Kidonakis, Phys. Rev. D 90, 014006 (2014) [arXiv:1405.7046 [hep-ph]]; 91, 031501(R) (2015) [arXiv:1411.2633 [hep-ph]]; 91, 071502(R) (2015) [arXiv:1501.01581 [hep-ph]].

[39] N. Kidonakis, Phys. Rev. Lett. 102, 232003 (2009) [arXiv:0903.2561 [hep-ph]]; Int. J. Mod. Phys. A 31, 1650076 (2016) [arXiv:1601.01666 [hep-ph]].

[40] J. Piclum and C. Schwinn, JHEP 1803, 164 (2018) [arXiv:1801.05788 [hep-ph]].

[41] CMS Collab., JHEP 1803, 115 (2018) [arXiv:1711.03143 [hep-ex]].

[42] ATLAS Collab., Eur. Phys. J. C 74, 3109 (2014) [Addendum: ibid. 76, 642 (2016)] [arXiv:1406.5375 [hep-ex]].

[43] CMS Collab., JHEP 1608, 029 (2016) [arXiv:1603.02303 [hep-ex]].

[44] ATLAS Collab., Eur. Phys. J. C 78, 487 (2018) [arXiv:1712.06857 [hep-ex]].

[45] ATLAS Collab., Phys. Lett. B 761, 136 (2016) [Erratum: ibid. 772, 879 (2017)] [arXiv:1606.02699 [hep-ex]].

[46] CMS Collab., JHEP 1709, 051 (2017) [arXiv:1701.06228 [hep-ex]].

[47] CDF Collab., Phys. Rev. D 64, 032002 (2001) [Erratum: ibid. 67, 119901 (2003)] [hepex/0101036].

[48] D0 Collab., Phys. Rev. D 67, 012004 (2003) [hep-ex/0205019].

[49] CDF and D0 Collab., Phys. Rev. D 89, 072001 (2014) [arXiv:1309.7570 [hep-ex]].

[50] CMS Collab., JHEP 1804, 060 (2018) [arXiv:1708.07638 [hep-ex]].

[51] ATLAS Collab., Eur. Phys. J. C 76, 538 (2016) [arXiv:1511.04716 [hep-ex]].

[52] CMS Collab., Eur. Phys. J. C 75, 542 (2015) [arXiv:1505.04480 [hep-ex]]. 